\title{
PERFIL NUTRICIONAL DE CRIANÇAS DE UMA ESCOLA EM BELÉM, PA
}

\author{
CHILDREN'S NUTRITIONAL PROFILE FROM A SCHOOL IN BELÉM, PA, BRAZIL

\section{Larissa do Socorro Ferreira dos Santos ${ }^{a}$, Sheila Cristina Martins e Silva ${ }^{b}$, Edson Marcos Leal Soares Ramos ${ }^{\mathrm{c}}$}

\author{
alara-sf@hotmail.com, bnutricsheilamartins@gmail.com, cramosedson@gmail.com
}

Universidade Federal do Pará - Belém (PA), Brasil

Data de recebimento do artigo: 23/09/2016

Data de aceite do artigo: 15/02/2017

\section{RESUMO}

Introduçáo: No Brasil, vêm ocorrendo mudanças no perfil nutricional da populaçáo que acometem não somente os adultos, mas também crianças e adolescentes, sendo perceptível o aumento do sobrepeso e da obesidade em idades cada vez mais precoces. Objetivo: Este estudo tem como objetivo traçar o perfil nutricional de crianças em idade pré-escolar matriculadas na Escola de Educação Infantil Jesus, localizada na cidade de Belém (PA). Metodologia: Trata-se de uma pesquisa de tipo transversal com crianças de 3 a 6 anos de idade, de ambos os sexos, realizada no período de novembro de 2015 a agosto de 2016. Foram coletado dados relativos a medidas de peso, estatura, idade e índice de massa corporal (IMC) das crianças. Posteriormente, a fim de determinar o diagnóstico nutricional, foram calculados os índices antropométricos de IMC por idade (IMC/I) e peso por estatura (P/E) em escore Z, segundo parâmetros estabelecidos em 2006/2007 pela Organização Mundial da Saúde (OMS). Resultados: Foram avaliadas 76 crianças, sendo $40(52,63 \%)$ do sexo masculino e $36(47,36 \%)$ do sexo feminino. Do total de crianças avaliadas, 67 (88,15\%) apesentaram diagnóstico nutricional adequado, 3 (3.94\%) apresentaram déficit e 6 \%7,89)) apresentaram excesso. Conclusão: Pôde-se concluir que a maioria das crianças obteve diagnóstico de eutrofia, o que pode ser atribuído ao acompanhamento nutricional e psicológico e à alimentação equilibrada oferecidos pela escola na qual estudam.

Palavras-chave: Estado nutricional; saúde da criança; pré-escolar; antropometria.

\section{ABSTRACT}

Introduction: In Brazil, several changes in the nutritional profile of the population have occurred, which affect not only the adult population, but also children and adolescents, with an increase in the overweight and obesity rates at increasingly early ages. Objective: The present study aimed at tracing the nutritional profile of children in preschool age, enrolled at the Jesus Early Childhood Education School, located in the city of Belém/PA. Methodology: The methodology is about a cross-sectional research conducted with children from3 to 6 years old, of both genders, from November 2015 to August 2016. Measurements of weight, stature, age and body mass index (BMI) were collected from these children. Afterwards, anthropometric indexes BMI/Age (BMI/A) and Weight/Stature (W/S) were calculated inz-score, according to 2006/2007 WHO, in order to determine the nutritional diagnosis. Results: The evaluation was made with 76 children, being $40(52.63 \%)$ of the male sex and $36(47.36 \%)$ of the female sex. From the total of evaluated children, $67(88.15 \%)$ demonstrated adequate nutritional diagnosis, 3 (3.94\%) deficit and $6(7.89 \%)$ excess. Conclusion: It was concluded that the majority of children had a eutrophic diagnosis, given that they have nutritional and psychological counseling, as well as a balanced diet from the school in which they study, a fact that contributes to an adequate nutritional status.

Keywords: Nutritional status; child health; preschool; anthropometry. 


\section{Introdução}

No Brasil, vêm ocorrendo mudanças no perfil nutricional da população que acometem não somente os adultos, mas também crianças e adolescentes, sendo perceptível o aumento do sobrepeso e da obesidade em idades cada vez mais precoces. Este fato tem sido motivo de preocupação e um desafio para profissionais de saúde e pesquisadores, visto que o aumento da obesidade na população infantil é observado em várias partes do mundo, gerando consequências negativas para a saúde e qualidade de vida não apenas nesse estágio, mas também na fase adulta ${ }^{1}$.

O acompanhamento da situação nutricional de crianças é um instrumento essencial para verificar as condições de saúde dessa população. As medidas antropométricas têm sido utilizadas desde o século XVIII como forma de avaliar a saúde, porém, só há menos de 40 anos foram normatizadas para avaliação do estado nutricional individual e de populaçóes. Dessa forma, as medidas de peso e estatura são consideradas de alta sensibilidade, principalmente durante a fase pré-escolar, para refletir variaçôes nas condiçóes nutricionais e, indiretamente, as influências do ambiente socioeconômico ${ }^{2}$.

Crianças estão cada vez mais vulneráveis ao desenvolvimento de obesidade e deficiência de micronutrientes, visto que estão consumindo cada vez mais alimentos ricos em gordura e açúcar, mas pobres em nutrientes e fibra alimentar. Além disso, estudos demonstram que as crianças optam mais por alimentos de alta densidade energética, principalmente por promoverem maior saciedade, prejudicando assim o consumo de frutas e verduras ${ }^{3}$.

$\mathrm{Na}$ fase pré-escolar, muitas crianças passam grande parte do dia em creches públicas, retornando para casa somente no final do dia ${ }^{4}$. Nesse contexto, a escola surge como ambiente privilegiado para o desenvolvimento de ações para melhoria das condições de saúde e estado nutricional das crianças, sendo um local estratégico para a concretização de iniciativas de promoção de saúde

O diagnóstico e a intervenção nutricional são cruciais no intuito de orientar pais, crianças e gestores de escolas sobre a importância de uma alimentação saudável, ajudando-os a promover, sempre que necessário, uma reeducaçáo alimentar com vistas a uma melhor qualidade de vida.

Diante disso, este estudo tem como objetivo traçar o perfil nutricional de crianças em idade pré-escolar de 3 a 6 anos, de ambos os sexos, matriculados em uma escola em Belém (PA).

\section{Metodologia}

O estudo foi realizado na Escola de Educação Infantil Jesus, vinculada à organização não governamental LBV
(Legião da Boa Vontade), localizada na cidade de Belém do Pará. A instituição atende 120 crianças de famílias de baixa renda, com idades entre 3 e 6 anos, em período integral, fornecendo educação básica, uniforme, material didático, alimentação balanceada com supervisão de nutricionista e atendimento social e psicológico, tudo de forma gratuita.

A pesquisa, de tipo transversal, foi realizada com crianças de 3 a 6 anos de idade, de ambos os sexos, no período de novembro de 2015 a agosto de 2016. O projeto foi aprovado pelo Comitê de Ética em Pesquisa do Instituto de Ciências da Saúde (ICS) da Universidade Federal do Pará (UFPA) sob o parecer $n^{\circ} 1.664 .815$.

Para participação na pesquisa, foram considerados os seguintes critérios: crianças cujos pais e/ou responsáveis assinaram o Termo de Consentimento Livre e Esclarecido (TCLE), que não possuíssem deficiências e que estiveram presentes no dia da coleta das medidas.

Foram coletados, na própria escola e em um único momento, dados de peso, estatura, idade e índice de massa corporal (IMC).

Para aferição do peso, foi utilizada a balança digital da marca Camry com capacidade máxima de pesagem de $150 \mathrm{~kg}$ e precisão de $100 \mathrm{~g}$. A criança foi posicionada no centro da plataforma do equipamento, em posição ereta, com o seu peso distribuído em ambos os pés (descalços). Os braços permaneciam estendidos ao longo do corpo, e as vestimentas eram leves, livres de qualquer objeto que interferisse na pesagem.

As medidas de estatura foram conferidas utilizando uma fita métrica inelástica de $150 \mathrm{~cm}$, fixada na parede para aferição da altura. As crianças estavam na mesma posição de aferição do peso descrita anteriormente. Depois de posicionadas, foi utilizada uma régua para marcar o ponto mais alto de suas cabeças, comprimindo os cabelos para melhor verificação da altura.

A idade foi obtida a partir da data de nascimento informada pela direçáo da escola. Já o IMC foi calculado a partir da fórmula preconizada pela Organização Mundial da Saúde (OMS): peso atual em quilogramas dividido pelo quadrado da altura em metros.

Para classificação do estado nutricional, foram utilizados os índices antropométricos de IMC por idade (IMC/I) e peso por estatura (P/E), avaliados segundo parâmetros da Organização Mundial da Saúde ${ }^{6}$, que define os seguintes pontos de corte: <escore-z -2 (baixo peso), escores-z entre $-2 \mathrm{e}+1$ (eutrofia), escore-z entre +1 e +3 (sobrepeso) e >escore- $\mathrm{z}+3$ (obesidade). $\mathrm{O}$ diagnóstico do estado nutricional foi realizado em escore-z com o auxílio dos softwares WHO Anthro e WHO Anthro Plus ${ }^{7}$. Para as crianças maiores de cinco anos, não se utilizou o índice $\mathrm{P} / \mathrm{E}$ por não ser recomendado pela OMS para esta faixa etária.

Os dados foram tabulados no software Microsoft Excel versão 2010. Para análise estatística, foi utilizado 
o teste exato de comparação entre duas proporçôes e o teste exato de Fisher para verificar a associação entre as variáveis qualitativas do estudo, utilizando-se o nível de significância de $5 \%(\mathrm{p}<0,05)$.

\section{Resultados e discussão}

Das 120 crianças matriculadas na escola em questão, apenas 76 atenderam aos critérios de inclusão previamente estabelecidos e puderam participar da pesquisa. Deste número, $40(52,63 \%)$ eram do sexo masculino e $36(47,36 \%)$ do sexo feminino. Na Tabela 1, apresenta-se o resultado referente às idades dessas crianças. Observou-se que a maioria da amostra avaliada $(38,15 \%)$ encontra-se na faixa dos 4 anos, sendo a minoria $(6,57 \%)$ pertencente à faixa dos 6 anos.

Tabela 1: Distribuição das crianças da Escola de Educação Infantil Jesus conforme gênero e idade

\begin{tabular}{lcc} 
Características & N & \% \\
\hline Gênero & 40 & 52,63 \\
\hline Masculino & 36 & 47,37 \\
\hline Feminino & 76 & 100 \\
\hline Total & & \\
\hline Idade & 19 & 25 \\
\hline 3 anos & 29 & 38,16 \\
\hline 4 anos & 23 & 30,26 \\
\hline 5 anos & 5 & 6,58 \\
\hline 6 anos & 76 & 100 \\
\hline Total & & \\
\hline
\end{tabular}

Nas tabelas 2 e 3 estão os resultados referentes à avaliação do estado nutricional: parâmetro IMC/I e parâmetro $\mathrm{P} / \mathrm{E}$, respectivamente. Observando as duas tabelas, pode-se observar a maior prevalência de IMC/I adequado $(88,16 \%)$ e $\mathrm{P} / \mathrm{E}$ adequado $(60,52 \%)$. Em relação ao déficit de IMC/I e P/E, observou-se apenas três $(3,95 \%)$ e duas crianças $(2,64 \%)$, respectivamente. No quesito excesso, obteve-se o resultado de seis $(7,90 \%)$ e uma criança $(1,32 \%)$.

Com o indicador IMC/I, capaz de diagnosticar crianças e adolescentes entre 0 e 19 anos, foi possível abranger todas as crianças inclusas na pesquisa $(n=76)^{6}$. Já no parâmetro $\mathrm{P} / \mathrm{E}$, em que a margem de avaliação nutricional se estende somente até os 5 anos de idade, pôde-se abranger apenas $n=49$ crianças.

Analisando-se o estado nutricional por idade, observou-se que as crianças de 4 anos apresentaram menor valor para sobrepeso $(n=0)$ e os maiores valores para eutrofia, tanto no índice IMC/I (35,52\%) quanto no índice P/E (35,53\%). Duas crianças dessa faixa de idade, porém, apresentaram desnutrição $(2,63 \%)$.

A partir do teste exato de comparação entre duas proporçóes e o teste exato de Fisher, pode-se perceber que o estado nutricional das crianças não apresentou diferenças significativas com relação às variáveis gênero $e$ idade, uma vez que valor de $\mathrm{p}$ foi menor que o nível de significância (5\%).

Cabe observar que as crianças participantes vivem em condição de vulnerabilidade econômica em função da baixa renda dos pais ou responsáveis, fato que pode ser inferido com base nos quesitos de seleção para matrícula na Escola de Educação Infantil Jesus. Essa vulnerabilidade náo teve impacto no estado nutricional das crianças, que em sua maioria apresentaram índices adequados. Outros estudos, porém, comprovam o contrário, demonstrando a associação entre baixa renda familiar e alta prevalência de desnutrição e insegurança alimentar ${ }^{8}$, em relação apontada também pela pesquisa de Cabral et al. ${ }^{9}$, que, ao avaliarem beneficiários do Programa Bolsa Família de Maceió (AL), encontraram alta ocorrência de desnutrição em crianças (18\%) e adolescentes (20\%).

Outro ponto a ser observado foi a permanência em tempo integral nas escolas, o que pode justificar o estado nutricional eutrófico apresentado pela maioria das crianças avaliadas, confirmando tendência verificada também em outros trabalhos ${ }^{10}$. Segundo a Resolução $\mathrm{n}^{\circ} 38$ do Fundo Nacional de Desenvolvimento da Educação $^{11}$ (FNDE) de julho de 2009, no mínimo 70\% das necessidades nutricionais diárias de alunos matriculados em escolas de período integral devem ser supridas pelo cardápio ofertado.

A merenda escolar tem como principal objetivo suprir (ainda que parcialmente) as necessidades nutricionais dos alunos, promovendo o bem-estar, formando bons hábitos alimentares, melhorando a capacidade de aprendizagem e mantendo a criança na escola. Sendo assim, é de fundamental importância a análise do valor nutricional das merendas, levando-se em conta tanto a qualidade quanto a quantidade dos alimentos oferecidos $^{12}$.

Diversos fatores influenciam a saúde e o crescimento das crianças, entre os quais se destaca a alimentação saudável, que garante um adequado crescimento, evita o excesso de peso e previne carências nutricionais ${ }^{13}$.

Analisando os dados do Sistema de Vigilância Alimentar e Nutricional (SISVAN) ${ }^{14}$ do ano de 2016, abrangendo a região de Belém (PA), observou-se, em um total de 101 crianças na faixa etária de 2 a 5 anos de idade, o resultado de $7,16 \%$ para magreza, $64,4 \%$ para eutrofia, $15,99 \%$ para risco de sobrepeso e $12,45 \%$ para excesso de peso. Comparando esses 
dados com os resultados deste estudo, verificam-se números relativamente semelhantes no que diz respeito ao estado nutricional eutrófico. Nota-se, porém, grande prevalência de risco de sobrepeso e excesso de peso, tendência que tem se apresentado também em estudos como o de Romagna et al. ${ }^{15}$, que, ao avaliarem 272 crianças e adolescentes, observaram a prevalência de excesso de peso em 31,7\%.

Tabela 2: Avaliação do estado nutricional das crianças da Escola de Educação Infantil Jesus através do indicador IMC/I

\begin{tabular}{|c|c|c|c|c|c|}
\hline \multirow[b]{2}{*}{ Variável } & \multirow[b]{2}{*}{ Categoria } & \multicolumn{4}{|c|}{ IMC/I } \\
\hline & & $\begin{array}{l}\text { Déficit } \\
\text { (N/\%) }\end{array}$ & $\begin{array}{c}\text { Adequado } \\
\text { (N/\%) }\end{array}$ & $\begin{array}{c}\text { Excesso } \\
(\mathrm{N} / \%)\end{array}$ & $\begin{array}{c}\text { Total } \\
(\mathrm{N} / \%)\end{array}$ \\
\hline \multirow{4}{*}{ Gênero } & Masculino & $1(1,32)$ & $35(46,05)$ & $4(5,26)$ & $40(52,63)$ \\
\hline & Feminino & $2(2,63)$ & $32(42,11)$ & $2(2,63)$ & $36(47,37)$ \\
\hline & Total & $3(3,95)$ & $67(88,16)$ & $6(7,89)$ & $76(100,00)$ \\
\hline & P - Valor* & & $\mathrm{p}=0,000$ & & \\
\hline \multirow{6}{*}{ Idade } & 3 anos & $0(0,00)$ & $18(23,68)$ & $1(1,32)$ & $19(25,00)$ \\
\hline & 4 anos & $2(2,63)$ & $27(35,52)$ & $0(0,00)$ & $29(38,15)$ \\
\hline & 5 anos & $0(0,00)$ & $19(25,00)$ & $4(5,26)$ & $23(30,26)$ \\
\hline & 6 anos & $1(1,32)$ & $3(3,95)$ & $1(1,32)$ & $5(6,59)$ \\
\hline & Total & $3(3,95)$ & $67(88,15)$ & $6(7,90)$ & $76(100,00)$ \\
\hline & P - Valor* & & $\mathrm{p}=0,000$ & & \\
\hline
\end{tabular}

Fonte: Protocolo de pesquisa, 2016.

*Teste exato de comparação entre duas proporçóes e o teste exato de Fisher.

Tabela 3: Avaliação do estado nutricional das crianças da Escola de Educação Infantil Jesus através do indicador P/E

\begin{tabular}{|c|c|c|c|c|c|c|}
\hline \multirow[b]{2}{*}{ Variável } & \multicolumn{6}{|c|}{$\mathrm{P} / \mathrm{E}$} \\
\hline & Categoria & $\begin{array}{l}\text { Déficit } \\
\text { (N/\%) }\end{array}$ & $\begin{array}{c}\text { Adequado } \\
(\mathrm{N} / \%)\end{array}$ & $\begin{array}{c}\text { Excesso } \\
(\mathrm{N} / \%)\end{array}$ & $\begin{array}{c}\text { Náo Mensurado } \\
(\mathrm{N} / \%)\end{array}$ & $\begin{array}{l}\text { Total } \\
(\mathrm{N} / \%)\end{array}$ \\
\hline \multirow{4}{*}{ Gênero } & Masculino & $1(1,32)$ & $22(28,94)$ & $1(1,32)$ & $16(21,05)$ & $40(52,63)$ \\
\hline & Feminino & $1(1,32)$ & $24(31,58)$ & $0(0,00)$ & $11(14,47)$ & $36(47,37)$ \\
\hline & Total & $2(2,64)$ & $46(60,52)$ & $1(1,32)$ & $27(35,52)$ & $76(100,00)$ \\
\hline & P - Valor* & & $\mathrm{p}=0,001$ & & & \\
\hline \multirow{6}{*}{ Idade } & 3 anos & $0(0,00)$ & $18(23,68)$ & $1(1,32)$ & $0(0,00)$ & $19(25,00)$ \\
\hline & 4 anos & $2(2,63)$ & $27(35,53)$ & $0(0,00)$ & $0(0,00)$ & $29(38,16)$ \\
\hline & 5 anos & $0(0,00)$ & $1(1,32)$ & $0(0,00)$ & $22(28,94)$ & $23(30,26)$ \\
\hline & 6 anos & $0(0,00)$ & 0,00 & $0(0,00)$ & $5(6,58)$ & $5(6,58)$ \\
\hline & Total & $2(2,63)$ & $46(60,53)$ & $1(1,32)$ & $27(35,52)$ & $76(100,00)$ \\
\hline & P - Valor* & & $\mathrm{p}=0,003$ & & & \\
\hline
\end{tabular}

Fonte: Protocolo de pesquisa, 2016.

*Teste exato de comparação entre duas proporçóes e o teste exato de Fisher. 
Estudos do Instituto Brasileiro de Geografia e Estatística (IBGE) ${ }^{16}$ demonstram que, no Brasil, em um período de 30 anos, o número de crianças e adolescentes acima do peso subiu de $4 \%$ para $18 \%$ entre meninos e de $7,5 \%$ para $15,5 \%$ nas meninas.

A ocorrência de excesso de peso em crianças está cada vez mais elevada, tendo como fator principal a alimentação inadequada, seguida do sedentarismo. De acordo com Ronsani et al. ${ }^{17}$, o aumento da prevalência do sobrepeso e obesidade contribui para uma maior ocorrência de doenças crônicas não transmissíveis (DCNT). Tais doenças, por serem de longo período de duração, são as que mais demandam açóes, serviços e procedimentos de saúde, levando a uma sobrecarga do SUS.

Além disso, o sobrepeso também pode influenciar no desempenho escolar, conforme observado no trabalho de Izidoro et al. ${ }^{18}$, que, analisando a relação entre estado nutricional e desempenho escolar, revelaram que crianças com excesso de peso apresentaram os piores resultados nas tarefas de escrita e aritmética, fato que pode ser justificado pelos baixos níveis de prática de atividade física e, consequentemente, menor disposição para realizar as atividades diárias.

Conforme Alves et al. ${ }^{19}$, a ingestáo dietética exerce papel fundamental no crescimento, desenvolvimento e formaçáo de hábitos alimentares da criança. Uma alimentação de baixo valor energético pode levar à carência de nutrientes, causando sérias consequências, como debilitação do sistema imune, baixa estatura e diminuiçẫo do rendimento escolar. Por outro lado, uma alimentação excessivamente calórica pode levar ao surgimento de doenças crônicas não transmissíveis a médio e longo prazo.

A escola, por ser um espaço onde muitas pessoas passam grande parte do tempo, torna-se um ambiente favorável para o desenvolvimento de açóes para a promoção da saúde e formação de bons hábitos alimentares, atingindo os estudantes nas etapas mais influenciáveis da sua vida, seja na infância ou na adolescência ${ }^{20}$.

Boccaletto e Mendes ${ }^{13}$ afirmam que "as crianças adquirem seus hábitos em dois ambientes que podem e devem estar em permanente diálogo: a própria família e a escola"; por isso, o diagnóstico e a intervenção nutricional sáo cruciais no intuito de orientar pais, crianças e gestores de escolas sobre a importância de uma alimentação saudável, ajudando-os a promover a reeducação alimentar com vistas a uma melhor qualidade de vida.

\section{Conclusão}

Conclui-se que, por meio da avaliação do perfil nutricional das crianças pertencentes à Escola de Educação Infantil Jesus, obteve-se maior prevalência do diagnóstico de eutrofia, o que pode ser atribuído ao acompanhamento nutricional e psicológico e à alimentação equilibrada oferecidos aos alunos pela instituição de ensino.

\section{Referências}

1. Carvalho AP, Oliveira VB, Santos LC. Hábitos alimentares e práticas de educação nutricional: atenção a crianças de uma escola municipal de Belo Horizonte, Minas Gerais. Pediatria (São Paulo). 2010;32(1):20-7.

2. Pandolfi MM, Sandrini F, Oliveira MR, Arnmond JE, Zollner ACR, Juliano Y, et al. Desnutrição em escolares em região urbano-rural do extremo sul do município de São Paulo. Einstein. 2011;9(4 Pt1):508-13.

3. Valmórbida JL, Vitolo MR. Fatores associados ao baixo consumo de frutas e verduras entre pré-escolares de baixo nível socioeconômico. J Pediatr. 2014;90(5):464-71.

4. Silva MC, Capanema FD, Lamounier JA, Silva ACA, Oliveira BM, Rodrigues J. Perfil nutricional de crianças pré-escolares em creches públicas de Belo Horizonte - Minas Gerais beneficiárias ou não do Programa Bolsa Família. Perc Acad. 2014;4(7):88-104.

5. Schmitz BAS, Recine E, Cardoso GT, Silva JRM, Amorim NFA, Bernardon R, et al. A escola promovendo hábitos alimentares saudáveis: uma proposta metodológica de capacitaçáo para educadores e donos de cantina escolar. Cad Saúde Pública. 2008;24(2):s312-s322.

6. Brasil. Ministério da Saúde. Incorporação das curvas de crescimento da Organização Mundial da Saúde de 2006 e 2007 no SISVAN [Internet]. Brasília: Ministério da Saúde; 2007 [citado em 2016 jan 18]. Disponível em: http://bit. ly/21HN99o

7. World Health Organization. WHO Anthro (version 3.2.2, January 2011) and macros [Internet]. Geneva: World Health Organization; c2017 [citado em 2017 mar 9]. Disponível em: http://bit.ly/2n49r5G

8. Monteiro F, Schmidt ST, Costa IB, Almeida CCB, Matuda NS. Bolsa Família: insegurança alimentar e nutricional de crianças menores de cinco anos. Ciên Saúde Coletiva. 2014;19(5):1347-58.

9. Cabral MJ, Vieira KA, Sawaya AL, Florencio TMMT. Perfil socioeconômico, nutricional e de ingestão alimentar de beneficiários do Programa Bolsa Família. Estud Av. 2013;27(78):71-87.

10. Goulardt RMM, Banduk MLS, Taddei JAAC. Uma revisão das açóes de nutrição e do papel do nutricionista em creches. Rev Nutr. 2010;23(4):655-65.

11. Brasil. Ministério da Educação. Fundo nacional de desenvolvimento da educação. Resoluçâo/CD/FNDE no 38, de 16 de julho de 2009 [citado em 25 ago 2016]. Dispóe sobre o atendimento da alimentação escolar aos alunos da educação básica no Programa Nacional de Alimentação Escolar - PNAE. Disponível em: http://bit.ly/2n3joQO 
12. Pontes R, Slompo RB, Luz PA, Passoni CMS. Influência da merenda escolar no estado nutricional. Estado nutricional e merenda escolar. Cad Esc Saúde. 2014;4(1):64-77.

13. Boccaletto EMA, Mendes RT. Alimentação, atividade física e qualidade de vida dos escolares do município de Vinhedo/ SP. Campinas: IPES; 2009.

14. Brasil. Ministério da Saúde. Protocolos do sistema de vigilância alimentar e nutricional SISVAN. Brasília: Ministério da Saúde; 2014 [citado em 26 ago 2016]. Disponível em: http://bit.ly/2nji9sL

15. Romagna ES, Silva MCA, Ballardin PAZ. Prevalência de sobrepeso e obesidade em crianças e adolescentes de uma unidade básica de saúde em Canoas, Rio Grande do Sul, e comparação do diagnóstico nutricional entre os gráficos do CDC 2000 e da OMS 2006. Sci Med. 2010;20(3):228-31.

16. Brasil. Instituto Brasileiro de Geografia e Estatística. Pesquisa de orçamentos familiares, 2002- 2003: análise da disponibilidade domiciliar de alimentos e do estado nutricional de crianças e adolescentes no Brasil. Rio de Janeiro: IBGE; 2004 [citado em 27 ago 2016]. Disponível em: http://bit. ly/2naHBBp

17. Ronsani MM, Silva ST, Ribeiro RSV. Atuação do nutricionista na atenção básica à saúde. Rev Programa Resid Multiprofissional Atenção Básica. 2013;1(1):94-109.

18. Izidoro GSL, Santos JN, Oliveira TSC, Martins-Reis VO. A influência do estado nutricional no desempenho escolar. Rev Cefac. 2014;16(5):1541-7.

19. Alves MN, Muniz LC, Vieira MFA. Consumo alimentar entre crianças brasileiras de dois a cinco anos de idade: Pesquisa Nacional de Demografia e Saúde (PNDS), 2006. Ciênc Saúde Coletiva. 2013;18(11):3369-77.

20. Fernandes FM. Alimentação e nutrição entre escolares: caso dos alunos de uma escola do município, Vitória - ES [monografia]. Vitória: Universidade Veiga de Almeida; 2006.

Como citar este artigo:

Santos LSF, Silva SCM, Ramos EMLS. Perfil nutricional de crianças de uma escola em Belém, PA. Rev. Aten. Saúde. 2017;15(51):69-74. 